\title{
Effects of lithium on inflammatory and neurotrophic factors after an immune challenge in a lisdexamfetamine animal model of mania
}

\author{
Giovana Bristot, ${ }^{1,2}$ iD Bruna M. Ascoli, ${ }^{1,3}$ Ellen Scotton, ${ }^{1,3}$ Luiza P. Géa, ${ }^{1,4}$ Bianca Pfaffenseller, ${ }^{1}$ \\ Márcia Kauer-Sant'Anna ${ }^{1,2,3}$ iD \\ ${ }^{1}$ Laboratório de Psiquiatria Molecular, Hospital de Clínicas de Porto Alegre, Porto Alegre, RS, Brazil. ${ }^{2}$ Programa de Pós-Graduação em \\ Bioquímica, Universidade Federal do Rio Grande do Sul (UFRGS), Porto Alegre, RS, Brazil. ${ }^{3}$ Programa de Pós-Graduação em Psiquiatria \\ e Ciências do Comportamento, UFRGS, Porto Alegre, RS, Brazil. ${ }^{4}$ Programa de Pós-Graduação em Farmacologia e Terapêutica, UFRGS \\ Porto Alegre, RS, Brazil.
}

\begin{abstract}
Objective: To evaluate whether an animal model of mania induced by lisdexamfetamine dimesylate (LDX) has an inflammatory profile and whether immune activation by lipopolysaccharides (LPS) has a cumulative effect on subsequent stimuli in this model. We also evaluated the action of lithium ( $\mathrm{Li}$ ) on inflammatory and neurotrophic factors.

Methods: Adult male Wistar rats were subjected to an animal model of mania. After the open-field test, they were given LPS to induce systemic immune activation. Subsequently, the animals' blood was collected, and their serum levels of brain-derived neurotrophic factor and inflammatory markers (tumor necrosis factor [TNF]- $\alpha$, interleukin [IL]-6, IL-1 $\beta, \mathrm{IL}-10$, and inducible nitric oxide synthase [iNOS]) were measured.

Results: LDX induced hyperactivity in the animals, but no inflammatory marker levels increased except brain-derived neurotrophic factor (BDNF). Li had no effect on serum BDNF levels but prevented iNOS levels from increasing in animals subjected to immune activation.

Conclusion: Although Li prevented an LPS-induced increase in serum iNOS levels, its potential antiinflammatory effects in this animal model of mania were conflicting.
\end{abstract}

Keywords: Bipolar disorder; mania; lithium; inflammation; lisdexamfetamine dimesylate

\section{Introduction}

Bipolar disorder is a severe psychiatric disorder affecting $1-4 \%$ of the population. ${ }^{1}$ Bipolar disorder is also associated with high rates of medical comorbidities, ${ }^{2}$ such as autoimmune disorders, cardiovascular disease, and metabolic dysfunction. ${ }^{3}$ The connection between bipolar disorder and these comorbidities seems to involve multiple biological pathways, among which inflammation stands out as a pivotal pathophysiological mechanism.

A recent study by Leboyer et al. suggested that lowgrade inflammation may be present in bipolar patients, based on alterations in peripheral and central inflammatory markers, which could be associated with damage to the central nervous system (CNS) ${ }^{4}$ A meta-analysis of 30 studies found that pro-inflammatory, anti-inflammatory, and regulatory cytokines are activated in bipolar disorder, although their precise role in the pathophysiology of this mental disorder remains unknown. ${ }^{5}$ Inducible nitric oxide synthase (iNOS) is involved in the production of a great

Correspondence: Márcia Kauer-Sant'Anna, Hospital de Clínicas de Porto Alegre, Centro de Pesquisa Experimental, Laboratório de Psiquiatria Molecular, Rua Ramiro Barcelos, 2350, CEP 90035-903, Porto Alegre, RS, Brazil.

E-mail: mksantanna@gmail.com

Submitted Dec 13 2017, accepted Sep 28 2018, Epub Mar 072019. amount of nitric oxide (NO) for sustained periods of time and has been linked to inflammation and the potential damaging actions of NO. In a study by Savas et al., higher plasma NO levels were found in bipolar patients than healthy subjects. ${ }^{6}$

Lithium ( $\mathrm{Li}$ ), the gold standard treatment for bipolar disorder, has been implicated in immune system modulation since the pioneering work of Horrobin \& Lieb in the early 1980 s. $^{7}$ Since then, the effects of Li on inflammation have been investigated in a number of studies. One of them found fewer cytokine-secreting cells (interleukin [IL]-6, IL-10, IL-2, and interferon [IFN]- $\gamma$ ) in bipolar patients after chronic Li treatment, suggesting that Li may normalize immune activation. ${ }^{8}$

In addition to its effects on inflammation, $\mathrm{Li}$ acts on other biological pathways related to oxidative stress and neurotrophins, ${ }^{9}$ probably helping to delay illness progression. Neurotrophins are essential for CNS development and maintenance. Among them is the brain-derived neurotrophic factor (BDNF), which is widely distributed in the

How to cite this article: Bristot G, Ascoli BM, Scotton E, Géa LP, Pfaffenseller B, Kauer-Sant'Anna M. Effects of lithium on inflammatory and neurotrophic factors after an immune challenge in a lisdexamfetamine animal model of mania. Braz J Psychiatry. 2019;41:419-427. http://dx.doi.org/10.1590/1516-4446-2017-0001 
brain and is associated with synaptic transmission and plasticity. ${ }^{10}$ Serum BDNF levels are lower in bipolar patients during mood episodes than in euthymic patients and controls. ${ }^{11}$ Among other therapeutic actions, it has been suggested that $\mathrm{Li}$ up-regulates BDNF, contributing to a euthymic state. ${ }^{11}$

Ever since increased dopaminergic transmission was observed in manic episodes, dopaminergic drugs have been used in research to mimic the manic phase of bipolar disorder and enable preclinical studies on this illness. ${ }^{12}$ Initially, Frey et al. proposed an animal model of mania induced by amphetamine. ${ }^{13}$ More recently, lisdexamfetamine dimesylate (LDX), a prodrug approved for treating attention deficit hyperactivity disorder, has also been used for this purpose. ${ }^{14}$ Although manic episodes are more complex than simply augmenting dopaminergic transmission, these models are useful for investigating new antimanic medications ${ }^{15}$ and understanding how classical mood stabilizers such as Li exert their effects.

There is evidence that immune activation plays a role in the pathophysiology of bipolar disorder and that $\mathrm{Li}$ may present potential anti-inflammatory properties. Therefore, in this study we aimed to investigate 1) whether an LDX-induced animal model of mania exhibits an inflammatory profile; 2) whether systemic immune activation by lipopolysaccharides (LPS) administration enhances the inflammatory profile presumably caused by LDX; and 3) whether $\mathrm{Li}$ can control this inflammatory response. Furthermore, we evaluated BDNF levels to assess whether there is interplay between inflammatory mediators and this neurotrophic factor in this animal model.

\section{Methods}

\section{Reagents}

LDX (Vyvanse ${ }^{\circledR}$, Shire, USA), Li chloride (LiCl; 213233; Sigma-Aldrich, St. Louis, MO, USA), and LPS from Escherichia coli 0111: B4 (L2630; Sigma-Aldrich, St. Louis, MO, USA) were used. Preparation of the drug solutions was performed as follows: for LDX, one LDX pill (70 mg) was dissolved in $35 \mathrm{~mL}$ of saline and the solution was sonicated for 30 minutes; for $\mathrm{LiCl}, 47.5 \mathrm{mg}$ of $\mathrm{LiCl}$ was dissolved in $1 \mathrm{~mL}$ of saline, mixing with a magnetic stirrer until a homogeneous solution was obtained; for LPS, $5 \mathrm{mg}$ of LPS was dissolved in $1 \mathrm{~mL}$ of saline, stirring until a homogeneous solution was obtained. All reagents were freshly prepared prior to use.

\section{Animals}

Sixty-day-old male Wistar rats (weighing 250-350 g) were obtained from the Central Animal House of the Universidade Federal do Rio Grande do Sul, Porto Alegre, state of Rio Grande do Sul, Brazil. They were housed in groups of four per cage in standard polycarbonate rat cages under standardized environmental conditions: a 12-hour light/ dark cycle (lights on between 7:00 a.m. and 7:00 p.m.), controlled temperature $\left(22 \pm 1{ }^{\circ} \mathrm{C}\right)$, and food and water available ad libitum. All experimental procedures were approved by the ethics committee for animal research of the Hospital de Clínicas de Porto Alegre (protocol 150538) and were carried out in accordance with the eighth edition of the National Institutes of Health (NIH) Guide for the Care and Use of Laboratory Animals. All efforts were made to minimize the number of animals and their suffering.

\section{Treatments}

The animals received one daily administration of either LDX (10 mg/kg), to induce hyperactivity (a manic episode feature), or saline solution by gavage for 14 days (32 rats per group) early in the morning (group allocation was randomized and counterbalanced). Although we chose a previously validated dose and vehicle, they had only been used in a single previous study ${ }^{14}$ and the validation was based solely on behavioral parameters. On the eighth day of treatment, the animals in the LDX $(\operatorname{LDX}+)$ and saline (LDX-) groups were randomly divided into two subgroups (16 rats each) and received an intraperitoneal administration of either $\mathrm{Li}(47.5 \mathrm{mg} / \mathrm{kg})$ or saline twice a day: in the morning (after the oral administration of LDX/saline) and in the evening (just before lights out). This was done to reproduce management of an acute manic episode, having been previously proposed as a reversal model of mania. ${ }^{12,13}$ On the 14 th day, locomotor activity was measured 2 hours after the final oral administration (LDX or saline). Half of the animals from each subgroup (eight rats) were then randomly allocated to receive one single intraperitoneal administration of LPS (5 mg/ $/ \mathrm{kg})$ to induce immune activation and the other half (eight rats) did not. The animals were anesthetized (ketamine $80-100 \mathrm{mg} / \mathrm{kg}$; xylazine $5-10 \mathrm{mg} / \mathrm{kg}$ ) and their blood was drawn from the tail vein 6 hours after LPS administration on the 14th day. Since it was not possible to collect blood from all the animals, biochemical analyses were carried out using seven animals in each group. The blood of rats that did not receive an LPS injection (control groups) was collected simultaneously with that of the respective LPS groups (Figure 1). The rats were then subjected to deep anesthesia and were euthanized by decapitation. The blood collected from each animal was allowed to clot, and the serum was separated by centrifugation $(1,000 \mathrm{~g} / 10 \mathrm{~min}-$ utes). Serum samples were stored at $-80^{\circ} \mathrm{C}$ until assay.

\section{Locomotor activity: open-field test}

An open-field test was used to assess locomotor activity 2 hours after the final oral administration (LDX or saline). The test was performed in an $80 \mathrm{~cm}$ diameter field enclosed by a $50 \mathrm{~cm}$ high acrylic wall with an open top. The floor of the arena was divided into segments, and the internal space of the arena was empty. The animals were gently placed at the center of the apparatus, without a previous habituation period, to explore the arena for 5 minutes. We used a video camera connected to a computer with ANY-maze software to record the behavior of each animal. The videos were analyzed using the ANYmaze behavioral tracking software, which provided data on the number of crossings, the total distance traveled (to determine horizontal activity) and the number of rearings (to determine vertical activity). 


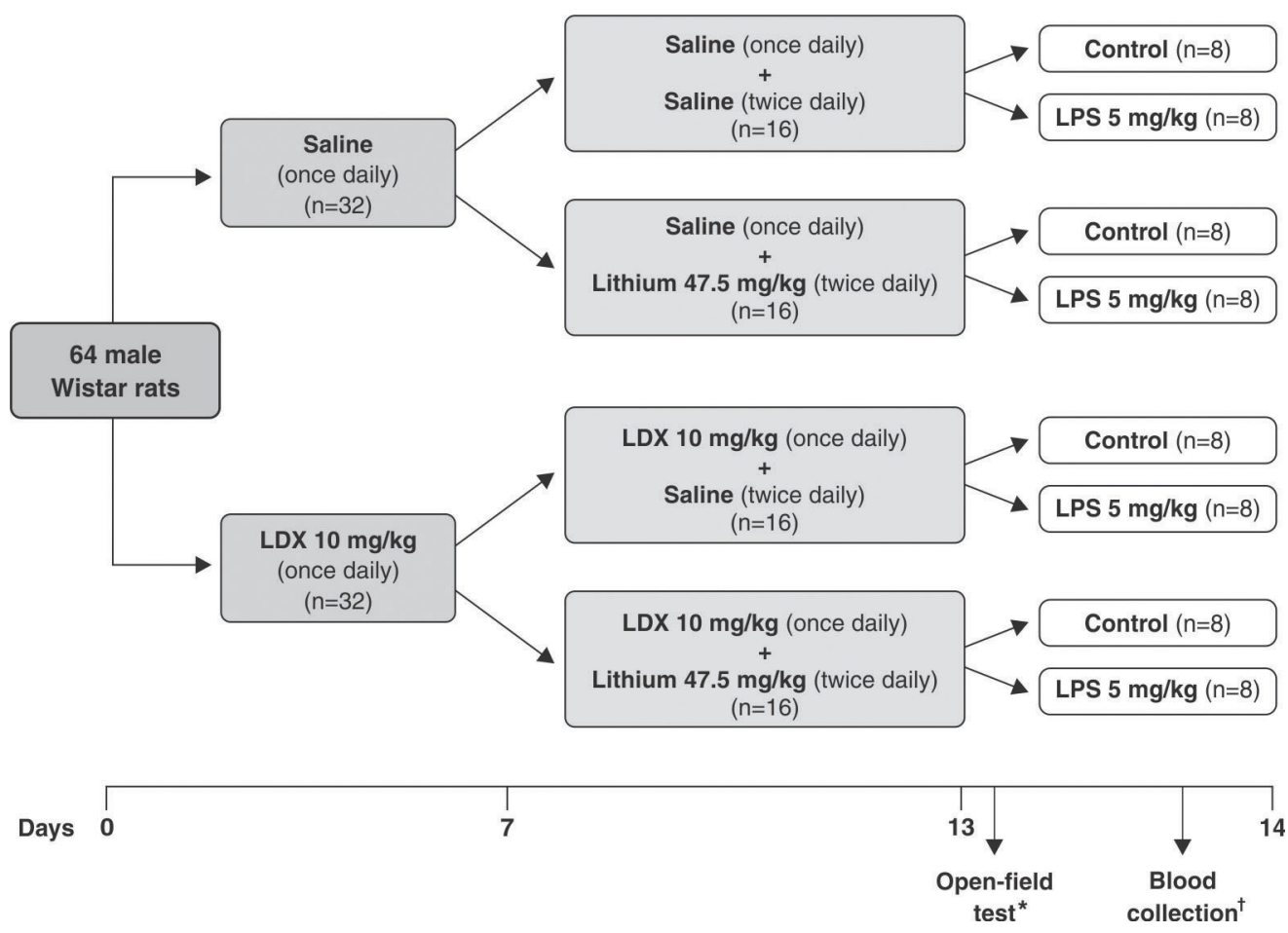

Figure 1 Overview of the experimental protocol: the blood of animals from the control groups was collected simultaneously with that of the respective LPS groups. LDX = lisdexamfetamine dimesylate; LPS = lipopolysaccharides. ${ }^{*}$ Two hours after the last LDX or saline administration; ${ }^{\dagger}$ six hours after LPS administration.

\section{Quantification of BDNF protein levels}

Serum BDNF levels were measured with sandwich ELISA using a commercial kit and following the manufacturer's instructions (Millipore, Massachusetts, USA). Microtiter plates (96-well flat-bottom) were incubated overnight at $4{ }^{\circ} \mathrm{C}$; the samples were diluted $1: 75$ with sample diluent in a standard curve ranging from 15.63 to $1,000 \mathrm{pg} / \mathrm{mL}$ of BDNF. Afterwards, the plates were washed four times with a wash buffer, followed by the addition of a biotinylated mouse anti-BDNF monoclonal antibody (diluted 1:1,000 with sample diluent) that had been incubated for 3 hours at room temperature (RT). After washing, the plates were incubated with streptavidin-horseradish peroxidase (HRP) conjugate solution (diluted 1:1,000 with sample diluent) for 1 hour at RT. After adding the substrate and stop solution, we determined the amount of BDNF (absorbance set at $450 \mathrm{~nm}$ ). The standard curve demonstrated a direct relationship between optical density (OD) and BDNF concentration.

\section{Quantification of iNOS levels}

iNOS serum levels were measured according to manufacturer's instructions (MyBioSource, California, USA) with an ELISA kit that uses the competitive enzyme immunoassay technique. We added standards or samples to the appropriate wells, then added phosphate buffered saline (PBS) ( $\mathrm{pH}$ 7.2) to the blank control well of a precoated plate. iNOS-HRP conjugate was added to each well (except the blank control well), and the plate was incubated for 1 hour at RT. After the incubation period, the wells were washed 5 times with diluted washing buffer solution. The HP substrate was pipetted and incubated for 15 minutes at RT. After a stop solution was added, the OD was determined spectrophotometrically at $450 \mathrm{~nm}$ using a microplate reader. The intensity of the color is inversely proportional to the iNOS concentration, since iNOS from the samples and iNOS-HRP conjugate compete for the anti-iNOS antibody-binding site. A standard curve was plotted by relating the intensity of the color (OD) to the concentration of standards, and the iNOS concentration in each sample was interpolated from this standard curve.

\section{Quantification of cytokine levels}

Tumor necrosis factor (TNF)- $\alpha$, IL-6, IL-1 $\beta$, and IL-10 serum levels were measured using a Milliplex Map Kit Rat cytokine/chemokine magnetic bead panel according to the manufacturer's instructions (Millipore, Massachusetts, USA). Standards or quality controls were added to the appropriate wells, and an assay buffer was added to background and sample wells. A matrix solution was then added to the background, standard, and control wells, and the samples were added to the appropriate wells. We pipetted beads into the wells and incubated the plate for 2 hours at RT. The plate was then washed twice with wash buffer, received detection antibodies, and was incubated with agitation on a plate shaker overnight at $4{ }^{\circ} \mathrm{C}$. After incubation, streptavidin conjugated with the fluorescent protein phycoerythrin was added, and the plate 
was incubated on a plate shaker for 30 minutes at RT. After washing to remove the unbound streptavidinphycoerythrin, sheath fluid was added to all wells, and the beads were resuspended on a plate shaker for 5 minutes. The beads (minimum of 50 beads per cytokine) were analyzed using a Luminex $200^{\mathrm{TM}}$ instrument, which monitored the spectral properties of the beads while simultaneously measuring the amount of fluorescence associated with phycoerythrin. Raw data (median fluorescent intensity) were analyzed using a five-parameter logistic method for calculating analyte concentrations in samples (Luminex xPONENT version 3.1).

\section{Statistical analyses}

Statistical analyses were performed using GraphPad Prism version 7.0. In all experiments, $p$-values under 0.05 were considered statistically significant. The results of the behavioral assessments are expressed as mean \pm standard error of the mean (SEM) of the distance traveled and the number of crossings and rearings. Behavioral data were checked for normality with the Shapiro-Wilk test and analyzed with two-way ANOVA followed by Bonferroni's test for post-hoc comparisons. The ShapiroWilk test also revealed that variables related to BDNF and iNOS measures were normally distributed. However, the residuals were not normally distributed for the cytokine data. Thus, we transformed data into a normal distribution by applying a natural $\log (\mathrm{In})$ transformation. The results of biochemical measurements are expressed as mean \pm SEM and were analyzed using three-way analysis of variance (ANOVA) followed by Bonferroni's test.

\section{Results}

\section{Locomotor activity}

The locomotor activity data obtained in the open-field test are presented in Figure 2. Two-way ANOVA showed an interaction between LDX and Li for total distance traveled $\left(n=16\right.$ per group; $\left.F_{1,60}=10.76 ; p=0.002\right)$ and the number of crossings ( $n=16$ per group; $F_{1,60}=7.995 ; p=0.006$ ). However, no interaction between these two factors was observed for the number of rearings ( $n=16$ per group; $\left.F_{1,60}=0.186 ; p=0.668\right)$. LDX significantly increased the total distance traveled ( $n=16$ per group; $F_{1,60}=45.55 ; p<$ 0.001 ), the number of crossings ( $n=16$ per group; $F_{1,60}=$ $46.7 ; p<0.001)$ and the number of rearings $(n=16$ per group; $F_{1,60}=10.02 ; p=0.002$ ). Li reduced the total distance traveled $\left(n=16\right.$ per group; $\left.F_{1,60}=75.45 ; p<0.001\right)$, the number of crossings ( $n=16$ per group; $F_{1,60}=68.2$; $p<0.001$ ), and the number of rearings ( $n=16$ per group; $\left.F_{1,60}=32.31 ; p<0.001\right)$. Post-hoc analysis showed that administering Li to LDX-treated animals reversed hyperactive behavior by reducing the total distance traveled $(p<0.001)$ and the number of crossings $(p<0.001)$.

\section{BDNF protein levels}

The serum BDNF protein levels of each group are presented in Figure 3. A three-way ANOVA test revealed that
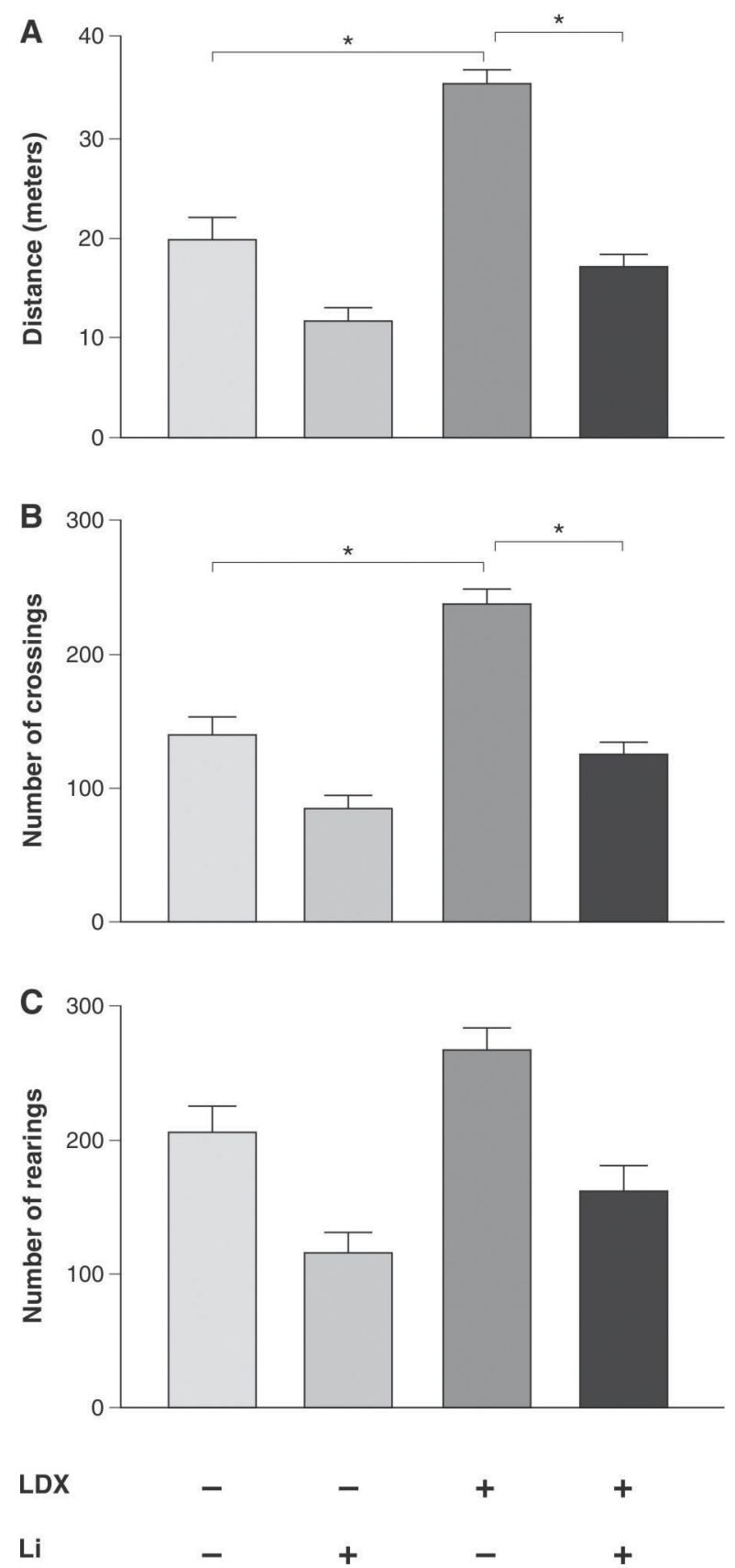

Figure 2 Open-field test: total distance traveled and the number of crossings and rearings after 7 days of treatment with LDX +7 days of LDX and Li. Bars represent mean \pm standard error of the mean. $n=16$ animals per group. LDX = lisdexamfetamine dimesylate; $\mathrm{Li}=$ lithium. ${ }^{*} \mathrm{p}<0.001$ according to two-way analysis of variance followed by Bonferroni's post-hoc test.

LDX treatment had a significant effect on BDNF protein levels ( $n=7$ per group; $F_{1,48}=205.1 ; p<0.001$ ). BDNF levels were higher in all groups that received LDX (LDX+ groups) than in LDX- groups, regardless of the presence of Li or LPS. This suggests that treatment with $\mathrm{Li}(\mathrm{n}=7$ per group; $\left.F_{1,48}=0.177 ; p=0.676\right)$ and LPS ( $n=7$ per group; $\left.\mathrm{F}_{1,48}=0.012 ; p=0.915\right)$ had no significant effect on BDNF levels. Furthermore, as can be seen in Table 1, 
three-way ANOVA revealed no interaction between the following analyzed factors: LDX vs. Li $(n=7$ per group; $\left.F_{1,48}=0.014 ; p=0.906\right)$; LDX vs. LPS $(n=7$ per group; $\left.F_{1,48}=0.134 ; p=0.716\right)$; Li vs. LPS ( $n=7$ per group; $F_{1,48}=$ $0.102 ; p=0.751)$; and LDX vs. Li vs. LPS ( $n=7$ per group;

$\left.F_{1,48}=0.521 ; p=0.474\right)$.

\section{iNOS levels}

Serum iNOS levels are shown in Figure 4. Although LDX $(p=0.781)$ had no influence on iNOS, Li $(p=0.002)$ and LPS ( $p<0.001$ ) had a significant effect on iNOS levels. We found no interactions between the following factors: LDX vs. Li ( $n=7$ per group; $\left.F_{1,48}=1.506 ; p=0.226\right)$; LDX vs. LPS ( $n=7$ per group; $\left.F_{1,48}=1.328 ; p=0.255\right)$; and $\mathrm{Li}$ vs. LPS ( $n=7$ per group; $\left.F_{1,48}=0.872 ; p=0.355\right)$. However, the interaction between LDX vs. Li vs. LPS $\left(n=7\right.$ per group; $\left.F_{1,48}=4.683 ; p=0.036\right)$ was significant.

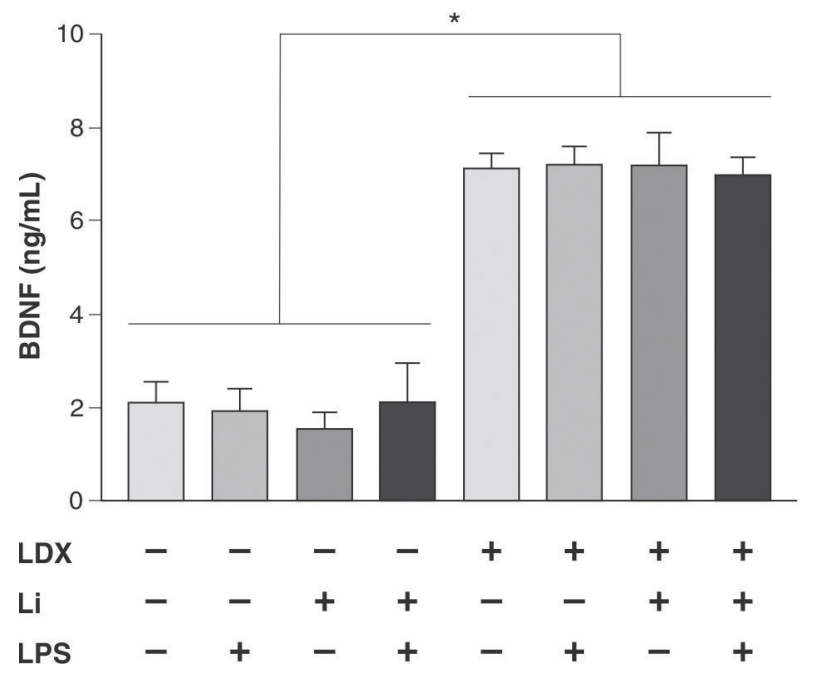

Figure 3 Serum BDNF protein levels after 7 days of treatment with LDX +7 days of LDX and $\mathrm{Li}$, and a single administration of LPS. The results are presented as concentration of BDNF $(\mathrm{ng} / \mathrm{mL})$. The bars represent mean \pm standard error of the mean. $\mathrm{n}=7$ animals per group. BDNF $=$ brain-derived neurotrophic factor; LDX = lisdexamfetamine dimesylate; $\mathrm{Li}=$ lithium; LPS $=$ lipopolysaccharides. ${ }^{*} p<0.001$ refers to the main effect of LDX, according to three-way analysis of variance.
The post-test for multiple comparisons between groups showed that the LDX-/Li-/LPS + group had a higher iNOS levels than LDX-/Li-/LPS- ( $p<0.001$ ) or LDX-/Li + /LPS + $(p<0.001)$, indicating that LPS increased iNOS levels and that $\mathrm{Li}$ could prevent this increase. No differences were found between groups that received LDX: the LDX + / Li-/LPS + group did not differ from the LDX+/Li-/LPSgroup ( $p=0.168$ ). This suggests that LDX helps prevent LPS-induced iNOS increases.

\section{Cytokine levels}

TNF- $\alpha$, IL-1 $\beta$, and IL-10 levels are shown in Figure 5. Three-way ANOVA revealed that neither LDX nor $\mathrm{Li}$ influenced TNF- $\alpha$, IL-1 $\beta$, and IL-10 levels. LPS, however, affected the levels of these cytokines ( $p<0.001)$. No significant interactions were observed between the factors, as shown in Table 1 . Since the kit was insufficiently

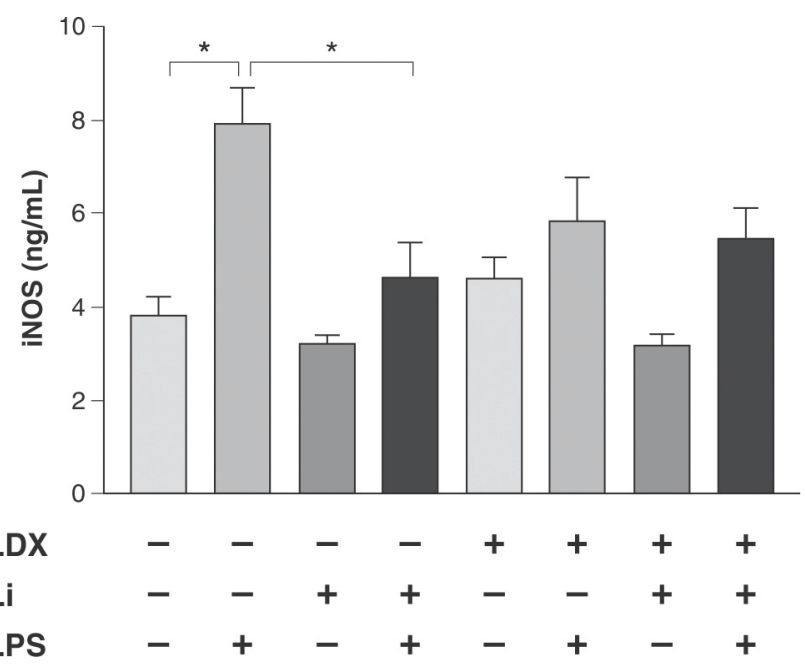

Figure 4 Serum iNOS levels after 7 days of treatment with LDX +7 days of LDX and $\mathrm{Li}$, and a single administration of LPS. The results are presented as concentration of iNOS $(\mathrm{ng} / \mathrm{mL})$. The bars represent mean \pm standard error of the mean. $n=7$ animals per group. iNOS = inducible nitric oxide synthase; LDX = lisdexamfetamine dimesylate; Li = lithium; LPS $=$ lipopolysaccharides. ${ }^{*} p<0.001$ according to three-way analysis of variance followed by Bonferroni's post-hoc test.

Table 1 The influence of LDX, Li, and LPS, as well as the interaction between these factors, on BDNF, iNOS, TNF- $\alpha$, IL-1 $\beta$, and IL-10 levels

\begin{tabular}{|c|c|c|c|c|c|c|c|c|c|c|}
\hline & \multicolumn{2}{|c|}{ BDNF } & \multicolumn{2}{|c|}{ iNOS } & \multicolumn{2}{|c|}{ TNF- $\alpha$} & \multicolumn{2}{|c|}{ IL-1 $\beta$} & \multicolumn{2}{|c|}{ IL-10 } \\
\hline & $\mathrm{F}_{1,48}$ & $p$-value & $F_{1,48}$ & $p$-value & $F_{1,48}$ & $p$-value & $F_{1,48}$ & p-value & $F_{1,48}$ & $p$-value \\
\hline LDX & 205.1 & $<0.001$ & 0.078 & 0.781 & 0.049 & 0.825 & 0.147 & 0.703 & 0.356 & 0.554 \\
\hline Li & 0.177 & 0.676 & 10.72 & 0.002 & 0.166 & 0.685 & 0.076 & 0.784 & 0.404 & 0.528 \\
\hline LPS & 0.012 & 0.915 & 27.44 & $<0.001$ & 111.82 & $<0.001$ & 20.375 & $<0.001$ & 36.485 & $<0.001$ \\
\hline LDX x Li & 0.014 & 0.906 & 1.506 & 0.226 & 2.713 & 0.105 & 0.729 & 0.397 & 2.226 & 0.142 \\
\hline LDX $x$ LPS & 0.134 & 0.716 & 1.328 & 0.255 & 2.213 & 0.143 & 3.235 & 0.078 & 1.883 & 0.176 \\
\hline Li x LPS & 0.102 & 0.751 & 0.872 & 0.355 & 3.458 & 0.068 & 0.93 & 0.34 & 3.738 & 0.059 \\
\hline LDX x Li x LPS & 0.521 & 0.474 & 4.683 & 0.036 & 2.612 & 0.112 & 0.744 & 0.393 & 0.524 & 0.473 \\
\hline
\end{tabular}

BDNF = brain-derived neurotrophic factor; IL = interleukin; iNOS = inducible nitric oxide synthase; LDX = lisdexamfetamine dimesylate; $\mathrm{Li}=$ lithium; LPS = lipopolysaccharides; TNF- $\alpha=$ tumor necrosis factor-alpha.

Values in bold font are significant. 

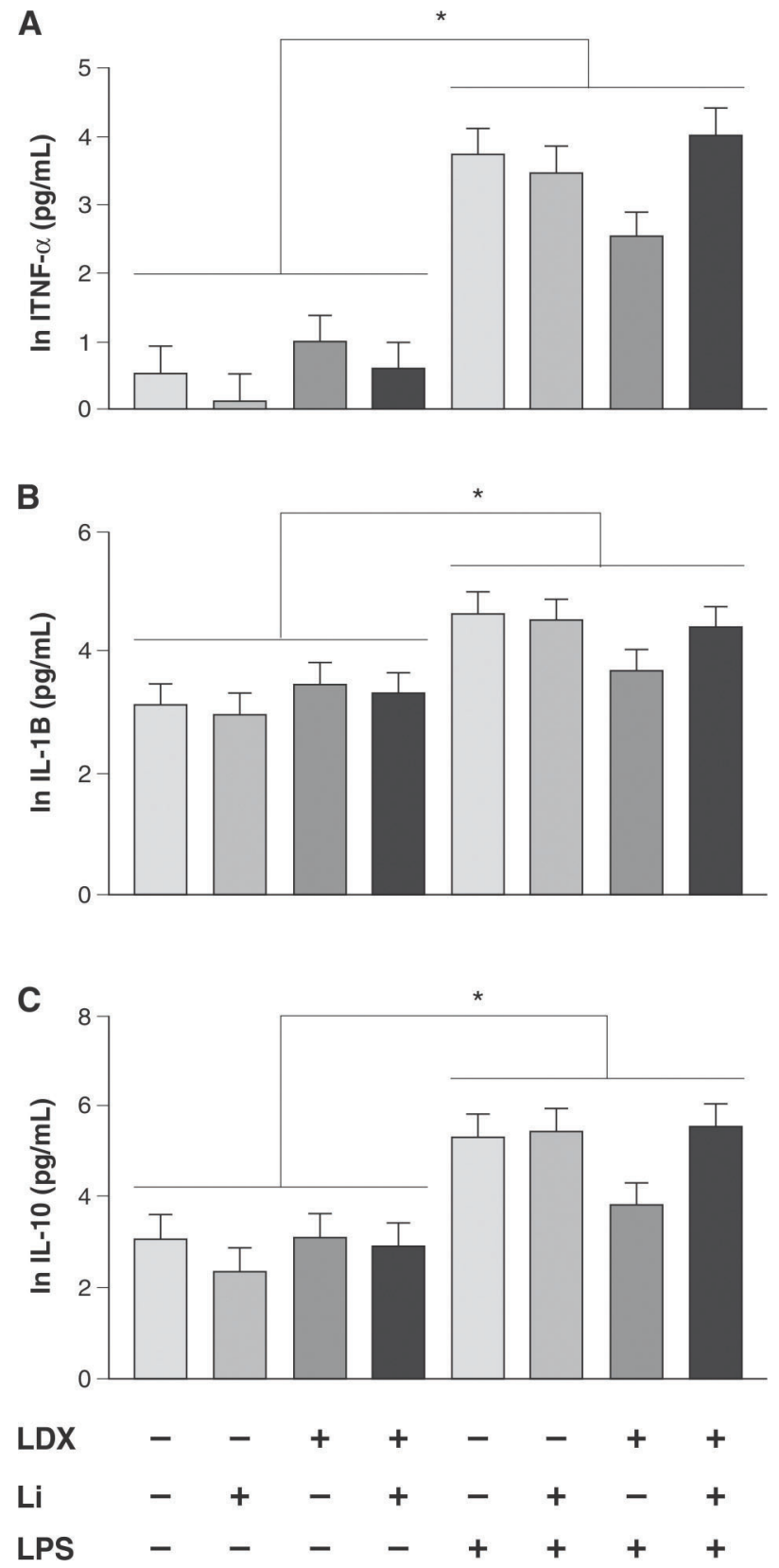

Figure 5 Serum TNF- $\alpha$, IL-1 $1 \beta$, and IL-10 levels after 7 days of treatment with LDX +7 days of LDX and Li, and a single administration of LPS. The results are presented as a natural $\log (\mathrm{In})$ of the concentration of cytokines $(\mathrm{pg} / \mathrm{mL})$. The bars represent mean \pm standard error of the mean. $n=7$ animals per group. IL = interleukin; LDX = lisdexamfetamine dimesylate; $\mathrm{Li}=$ lithium; LPS = lipopolysaccharides; TNF- $\alpha=$ tumor necrosis factor-alpha. ${ }^{*} p<0.001$ refers to the main effect of LPS, according to three-way analysis of variance.

sensitive to measure IL-6 $(73.2 \mathrm{pg} / \mathrm{mL})$ in the serum of animals that did not receive LPS, statistical analysis regarding IL-6 levels could not be carried out.

\section{Discussion}

This study aimed to investigate potential anti-inflammatory effects of $\mathrm{Li}$ and the interaction with BDNF levels in an animal model of mania with additional immune activation. Our results showed that LDX induced hyperlocomotion, the main hallmark of models of mania induced by psychostimulants, ${ }^{13,14}$ and increased rearing behavior, another feature found in such models. Moreover, we demonstrated that Li reversed the hyperactivity that LDX induced, which corroborates Macêdo et al. ${ }^{14}$ Although there are limitations to animal models of mania, this approach may be used to study specific features of the illness, understand specific biological pathways through which mood stabilizers act, and test potential new drugs for bipolar disorder. ${ }^{16,17}$

The inflammatory profile that was expected after LDX administration did not occur in our model, in contrast to a study by Valvassori et al. ${ }^{17}$ in which amphetamine increased inflammatory cytokine levels. Conversely, LDX prevented an increase in iNOS levels and tended to prevent an increase in inflammatory cytokine levels after an immune challenge (LPS administration). LPS is an endotoxin that induces sickness behavior by mimicking an infection caused by Gram-negative bacteria, which triggers the secretion of cytokines such as TNF- $\alpha$, IL-6, $\mathrm{IL}-1 \beta$, and IL- 10 by the immune system ${ }^{18}$ and increases expression of iNOS. ${ }^{19}$

Our study suggests that this occurred because LDX activates serine-threonine kinases such as cyclic adenosine monophosphate (cAMP)-dependent protein kinase A (PKA), protein kinase $C$ (PKC), and calmodulin kinases (CaMKs). These kinases promote phosphorylation and activation of the cAMP-responsive element binding protein (CREB), a transcriptional factor involved in many cellular processes, including cell survival, adaptive responses, synaptic plasticity, and immune responses. ${ }^{20}$ Once phosphorylated, CREB binds to coactivator CREB-binding protein (CBP). CBP can also bind to nuclear factor kappa light-chain enhancer in activated $B$ cells (NF-kB), which is a master regulator of the transcription of several genes involved in immune and inflammatory responses. ${ }^{21}$ Thus, CREB and NF-kB compete for limited amounts of CBP. When CREB is activated, the binding of CBP to CREB increases, while the binding of CBP to NF-kB decreases. This leads to a reduction in pro-inflammatory cytokines and iNOS expression, resulting in a substantial decline in the inflammatory process. ${ }^{20}$ This hypothesis agrees with the evidence that amphetamine increases CREB phosphorylation. ${ }^{22}$

LDX also increased serum BDNF levels in our study. This differs from a previous study that found lower BDNF levels in the hippocampus of rats subjected to this same model of mania. ${ }^{15}$ However, our results are in line with studies using animal models. Meredith et al. found higher BDNF expression in rat brain structures after repeated exposure to amphetamine. ${ }^{23}$ Methamphetamine also increased BDNF levels in the hippocampus ${ }^{24}$ and dorsal striatum $^{25}$ of rats. The occurrence of higher BDNF levels after a neurotoxic insult has been reported as a neuroprotective factor in prior studies. ${ }^{26,27}$ Based on these data, it could be speculated that the higher BDNF levels found in our model were an endogenous neuroprotective compensatory mechanism to the LDX neurotoxic insult. LDX might influence BDNF levels through CREB, since 
this transcription factor has also been implicated in the activation of BDNF gene expression. ${ }^{28}$

Therefore, the increase in endogenous BDNF we found in response to LDX may also be modulating the LPS-triggered immune activation, since rats administered with LDX tended to have lower cytokine levels. This hypothesis is based on a study by Jiang et al. which showed that BDNF was a potential inflammation modulator on the transcriptional and cellular levels. ${ }^{29}$

Contrary to expectations, LPS did not affect serum BDNF protein levels in any group. As an activator of NF-kB and stimulator of cytokine production, LPS was expected to reduce BDNF levels, as occurred in studies by Guan et al. and Schnydrig et al. in the brain structures of rats and mice, respectively. ${ }^{30,31}$ However, it should be noted that we measured serum BDNF protein levels 6 hours after LPS administration, while these studies analyzed BDNF expression in CNS structures and after a longer period: 7 hours $^{30}$ and within 1-6 days ${ }^{31}$ after LPS. Blood collection time was chosen based on a study showing that cytokine levels were still elevated 6 hours after LPS injection, ${ }^{32}$ although LPS-induced changes in serum BDNF protein levels appear at a longer latency. Moreover, BDNF levels were not affected by $\mathrm{Li}$ in our study, a result that differs from Frey et al. ${ }^{12}$ However, this may have been due to the short period (7 days) over which the animals received the drug, which is corroborated by Fukumoto et al. ${ }^{33}$ These authors found that chronic (14 to 28 days) but not acute ( 7 days) Li treatment increased BDNF expression in the hippocampus and frontal cortex of rats.

As expected, however, LPS increased the level of inflammatory mediators. In agreement with previous studies, we found elevated TNF- $\alpha, \mathrm{IL}-1 \beta, \mathrm{IL}-10,{ }^{32}$ and iNOS levels ${ }^{34}$ in the animals that received LPS. IL- $1 \beta$ and TNF- $\alpha$ increase after LPS injection because they are involved in the acute phase response of inflammation, while IL-10, an anti-inflammatory cytokine, is up-regulated to control the duration and intensity of the inflammatory response. ${ }^{32}$

Some studies have shown that Li has anti-inflammatory properties due to its ability to reduce inflammatory markers. ${ }^{35,36}$ Nonetheless, in our study, a therapeutic concentration of $\mathrm{Li}$ only prevented increased iNOS levels and had no effect on cytokine levels after the LPS challenge. In an in vitro experiment, Nahman et al. observed that $\mathrm{Li}$ reduced iNOS expression and TNF- $\alpha$ and IL-1 $\beta$ secretion, but these results were only obtained with an extra-therapeutic concentration of the drug. ${ }^{36}$ Evidence suggests that $\mathrm{Li}$ exerts its potential anti-inflammatory effects by inhibiting glycogen synthase kinase-3 $\beta$ (GSK$3 \beta$ ), which might reduce the activation of NF-kB and lead to a decrease in inflammatory mediator secretion and suppression of iNOS activity. ${ }^{37}$

It should be pointed out that we observed face and predictive validities in this animal model of mania, since the animals' behavior (hyperactivity) was similar to symptoms seen in bipolar patients (face validity) and Li treatment reversed the behavioral changes caused by LDX (predictive validity). However, construct validity was not observed, considering that the known biological basis of bipolar disorder was not reproduced. LDX did not affect inflammatory cytokine levels and caused an increase in serum BDNF levels, quite unlike what is observed in bipolar patients during mood episodes, such as increased cytokine levels ${ }^{38}$ and reduced BDNF levels. ${ }^{39}$ To a certain extent, it was expected that the model would reproduce only part of the complex pathophysiology involved in the illness. Nevertheless, based on the results obtained from this study, it seems that this animal model was inappropriate to evaluate the inflammatory changes commonly seen in bipolar patients. The difficulty in reproducing these features may be related to the drug used to induce the model of mania and to the LDX treatment time. Moreover, other limitations should be addressed, including our use of animals that did not receive vehicle injection as a control group for those that received LPS injection. In fact, this decision may have maximized the differences between LPS groups and their respective controls, considering that the injection itself may cause tissue damage. However, this does not appear to have been the case, considering that animals from non-LPS groups received previous intraperitoneal injections and such intervention did not influence any measured parameter. Finally, we should mention that the method that we used to detect BDNF does not distinguish between mature BDNF and proBDNF molecules, and further studies will be necessary to properly elucidate the results observed in our work.

It is important to emphasize that contrary to our initial hypothesis, LDX neither induced inflammation nor potentialized LPS-induced inflammatory activation. This might be considered the major limitation of our study, since we could not arrive at any conclusion about the protective potential of $\mathrm{Li}$ on the cumulative effect of LDX and LPS. Instead, LDX reduced inflammatory response after LPS, an unexpected and potentially interesting finding, given that LDX is not only used in animal models, but also as an effective and approved treatment for psychiatric disorders.

Our study's findings suggest that LDX induced hyperactivity, but did not induce an inflammatory profile in rats. Therefore, results about the possible anti-inflammatory effects of $\mathrm{Li}$ in this model of mania were conflicting. Li neither influenced BDNF serum levels nor prevented an increase in cytokines after the LPS challenge; however, it did prevent increased iNOS levels due to systemic immune activation, indicating that it might have some anti-inflammatory properties. Furthermore, animals that received $L D X$ showed an increase in serum BDNF, which may have occurred as an endogenous neuroprotective response to neurotoxic insult from LDX. This suggests that BDNF may have been essential in preventing increased iNOS levels in response to immune activation. Further experiments are needed to understand downstream immune and neurotrophic changes that may be associated with LDX, LPS, and Li.

\section{Acknowledgements}

The authors would like to thank the Fundo de Incentivo à Pesquisa - Hospital de Clínicas de Porto Alegre (FIPEHCPA), the Conselho Nacional de Desenvolvimento Científico e Tecnológico (CNPq), and the Coordenação 


\section{de Aperfeiçoamento de Pessoal de Nível Superior (CAPES) for funding support.}

MK-S has received research grants from CNPqNational Science and Technology Institute for Translational Medicine (INCT-TM), CNPq Universal, CAPES, the Stanley Medical Research Institute (SMRI), and the National Alliance for Research in Schizophrenia and Affective Disorders (NARSAD) Young Investigator Award.

\section{Disclosure}

MK-S has received research grants from Astra-Zeneca and Lilly. The other authors report no conflicts of interest.

\section{References}

1 Saunders KE, Geddes JR. The management of bipolar disorder. Br J Hosp Med (Lond). 2016;77:175-9.

2 Forty L, Ulanova A, Jones L, Jones I, Gordon-Smith K, Fraser C, et al. Comorbid medical illness in bipolar disorder. Br J Psychiatry. 2014;205:465-72.

3 Crump C, Sundquist K, Winkleby MA, Sundquist J. Comorbidities and mortality in bipolar disorder: a Swedish national cohort study. JAMA Psychiatry. 2013;70:931-9.

4 Leboyer M, Oliveira J, Tamouza R, Groc L. Is it time for immunopsychiatry in psychotic disorders? Psychopharmacology (Berl). 2016;233:1651-60.

5 Modabbernia A, Taslimi S, Brietzke E, Ashrafi M. Cytokine alterations in bipolar disorder: a meta-analysis of 30 studies. Biol Psychiatry. 2013;74:15-25.

6 Savaș HA, Herken H, Yürekli M, Uz E, Tutkun H, Zoroğlu SS, et al. Possible role of nitric oxide and adrenomedullin in bipolar affective disorder. Neuropsychobiology. 2002;45:57-61.

7 Horrobin DF, Lieb J. A biochemical basis for the actions of lithium on behaviour and on immunity: relapsing and remitting disorders of inflammation and immunity such as multiple sclerosis or recurrent herpes as manic-depression of the immune system. Med Hypotheses. 1981;7:891-905

8 Boufidou F, Nikolaou C, Alevizos B, Liappas IA, Christodoulou GN. Cytokine production in bipolar affective disorder patients under lithium treatment. J Affect Disord. 2004;82:309-13.

9 Goldstein BI, Kemp DE, Soczynska JK, Mclntyre RS. Inflammation and the phenomenology, pathophysiology, comorbidity, and treatment of bipolar disorder: a systematic review of the literature. J Clin Psychiatry. 2009;70:1078-90.

10 Shaltiel G, Chen G, Manji HK. Neurotrophic signaling cascades in the pathophysiology and treatment of bipolar disorder. Curr Opin Pharmacol. 2007;7:22-6.

11 Tunca Z, Ozerdem A, Ceylan D, Yalcin Y, Can G, Resmi H, et al. Alterations in BDNF (brain derived neurotrophic factor) and GDNF (glial cell line-derived neurotrophic factor) serum levels in bipolar disorder: the role of lithium. J Affect Disord. 2014;166:193-200.

12 Berk M, Dodd S, Kauer-Sant'anna M, Malhi GS, Bourin M, Kapczinski $F$, et al. Dopamine dysregulation syndrome: implications for a dopamine hypothesis of bipolar disorder. Acta Psychiatr Scand Suppl. 2007;434:41-9.

13 Frey BN, Andreazza AC, Ceresér KM, Martins MR, Valvassori SS, Réus GZ, et al. Effects of mood stabilizers on hippocampus BDNF levels in an animal model of mania. Life Sci. 2006;79:281-6.

14 Macêdo DS, de Lucena DF, Queiroz Al, Cordeiro RC, Araújo MM, Sousa FC, et al. Effects of lithium on oxidative stress and behavioral alterations induced by lisdexamfetamine dimesylate: relevance as an animal model of mania. Prog Neuropsychopharmacol Biol Psychiatry. 2013;43:230-7

15 de Souza GC, Gomes JA, de Gois Queiroz Al, de Araujo MM, Cavalcante LM, Machado Mde J, et al. Preclinical evidences for an antimanic effect of Carvedilol. Neural Plast. 2015;2015:692541.

16 Resende WR, Valvassori SS, Reus GZ, Varela RB, Arent CO, Ribeiro KF, et al. Effects of sodium butyrate in animal models of mania and depression: implications as a new mood stabilizer. Behav Pharmacol. 2013;24:569-79.

17 Valvassori SS, Tonin PT, Varela RB, Carvalho AF, Mariot E, Amboni $\mathrm{RT}$, et al. Lithium modulates the production of peripheral and cerebral cytokines in an animal model of mania induced by dextroamphetamine. Bipolar Disord. 2015;17:507-17.

18 Moraes MM, Galvao MC, Cabral D, Coelho CP, QueirozHazarbassanov N, Martins MF, et al. Propentofylline prevents sickness behavior and depressive-like behavior induced by lipopolysaccharide in rats via neuroinflammatory pathway. PLoS One. 2017; 12:e0169446.

19 Saha RN, Pahan K. Regulation of inducible nitric oxide synthase gene in glial cells. Antioxid Redox Signal. 2006;8:929-47.

20 Wen AY, Sakamoto KM, Miller LS. The role of the transcription factor CREB in immune function. J Immunol. 2010;185:6413-9.

21 Lawrence T. The nuclear factor NF-kappaB pathway in inflammation. Cold Spring Harb Perspect Biol. 2009;1:a001651.

22 Choe ES, Chung KT, Mao L, Wang JQ. Amphetamine increases phosphorylation of extracellular signal-regulated kinase and transcription factors in the rat striatum via group I metabotropic glutamate receptors. Neuropsychopharmacology. 2002;27:565-75.

23 Meredith GE, Callen S, Scheuer DA. Brain-derived neurotrophic factor expression is increased in the rat amygdala, piriform cortex and hypothalamus following repeated amphetamine administration. Brain Res. 2002;949:218-27.

24 McFadden LM, Vieira-Brock PL, Hanson GR, Fleckenstein AE. Methamphetamine self-administration attenuates hippocampal serotonergic deficits: role of brain-derived neurotrophic factor. Int $\mathrm{J}$ Neuropsychopharmacol. 2014;17:1315-20.

25 Krasnova IN, Chiflikyan M, Justinova Z, McCoy MT, Ladenheim B, Jayanthi $S$, et al. CREB phosphorylation regulates striatal transcriptional responses in the self-administration model of methamphetamine addiction in the rat. Neurobiol Dis. 2013;58:132-43.

26 Braun AA, Herring NR, Schaefer TL, Hemmerle AM, Dickerson JW, Seroogy KB, et al. Neurotoxic (+)-methamphetamine treatment in rats increases brain-derived neurotrophic factor and tropomyosin receptor kinase $B$ expression in multiple brain regions. Neuroscience. 2011;184:164-71.

27 Martinez-Serrano A, Bjorklund A. Protection of the neostriatum against excitotoxic damage by neurotrophin-producing, genetically modified neural stem cells. J Neurosci. 1996;16:4604-16.

28 Tao X, Finkbeiner S, Arnold DB, Shaywitz AJ, Greenberg ME. Ca2 + influx regulates BDNF transcription by a CREB family transcription factor-dependent mechanism. Neuron. 1998;20:709-26.

29 Jiang Y, Wei N, Zhu J, Lu T, Chen Z, Xu G, et al. Effects of brainderived neurotrophic factor on local inflammation in experimental stroke of rat. Mediators Inflamm. 2010;2010:372423.

30 Guan Z, Fang J. Peripheral immune activation by lipopolysaccharide decreases neurotrophins in the cortex and hippocampus in rats. Brain Behav Immun. 2006;20:64-71.

31 Schnydrig S, Korner L, Landweer S, Ernst B, Walker G, Otten U et al. Peripheral lipopolysaccharide administration transiently affects expression of brain-derived neurotrophic factor, corticotropin and proopiomelanocortin in mouse brain. Neurosci Lett. 2007;429:69-73.

32 Biesmans S, Meert TF, Bouwknecht JA, Acton PD, Davoodi N, De Haes $P$, et al. Systemic immune activation leads to neuroinflammation and sickness behavior in mice. Mediators Inflamm. 2013;2013: 271359.

33 Fukumoto T, Morinobu S, Okamoto Y, Kagaya A, Yamawaki S. Chronic lithium treatment increases the expression of brain-derived neurotrophic factor in the rat brain. Psychopharmacology (Berl). 2001;158:100-6.

34 Jung YS, Park JH, Kim H, Kim SY, Hwang JY, Hong KW, et al. Probucol inhibits LPS-induced microglia activation and ameliorates brain ischemic injury in normal and hyperlipidemic mice. Acta Pharmacol Sin. 2016;37:1031-44.

35 Albayrak A, Halici Z, Polat B, Karakus E, Cadirci E, Bayir Y, et al. Protective effects of lithium: a new look at an old drug with potential antioxidative and anti-inflammatory effects in an animal model of sepsis. Int Immunopharmacol. 2013;16:35-40.

36 Nahman S, Belmaker RH, Azab AN. Effects of lithium on lipopolysaccharide-induced inflammation in rat primary glia cells. Innate Immun. 2012;18:447-58. 
37 Wang HM, Zhang T, Li Q, Huang JK, Chen RF, Sun XJ. Inhibition of glycogen synthase kinase-3beta by lithium chloride suppresses 6-hydroxydopamine-induced inflammatory response in primary cultured astrocytes. Neurochem Int. 2013;63:345-53.

38 Brietzke E, Stertz L, Fernandes BS, Kauer-Sant'anna M, Mascarenhas M, Escosteguy Vargas A, et al. Comparison of cytokine levels in depressed, manic and euthymic patients with bipolar disorder. J Affect Disord. 2009;116:214-7.

39 de Oliveira GS, Cereser KM, Fernandes BS, Kauer-Sant'Anna M, Fries GR, Stertz L, et al. Decreased brain-derived neurotrophic factor in medicated and drug-free bipolar patients. J Psychiatr Res. 2009; 43:1171-4. 